\title{
Effect of Mediterranean Diet and Antioxidant Formulation in Non-Alcoholic Fatty Liver Disease: A Randomized Study
}

\author{
Ludovico Abenavoli ${ }^{1, *}$ (D), Marta Greco ${ }^{1}$ (D), Natasa Milic ${ }^{2}$, Francesca Accattato ${ }^{1}$, \\ Daniela Foti ${ }^{1}{ }^{\mathbb{D}}$, Elio Gulletta ${ }^{1}$ and Francesco Luzza ${ }^{1}$ \\ 1 Department of Health Sciences, University “Magna Græcia”, 88100 Catanzaro, Italy; \\ marta.greco@unicz.it (M.G.); francescaaccattato@libero.it (F.A.); foti@unicz.it (D.F.); \\ gulletta@unicz.it (E.G.); luzza@unicz.it (F.L.) \\ 2 Department of Pharmacy, University of Novi Sad, 21000 Novi Sad, Serbia; milnat@libero.it \\ * Correspondence: 1.abenavoli@unicz.it; Tel.: +39-096-1369-4387; Fax: +39-096-175-4220
}

Received: 3 July 2017; Accepted: 8 August 2017; Published: 12 August 2017

\begin{abstract}
Non-alcoholic fatty liver disease (NAFLD) is the most common liver disease worldwide, characterized by liver fatty acid accumulation and fibrosis, not due to excessive alcohol consumption. Notably, nutritional habits have been reported to be implicated in the onset and severity of the hepatic damage, while the Mediterranean diet has shown beneficial effects on NAFLD. Free radicals and oxidative stress were suggested to be involved in the pathogenesis and progression of NAFLD, and several data highlighted the efficacy of antioxidant supplementation in its treatment. The aim of this study was to compare the effects of the Mediterranean diet, with or without an antioxidant complex supplement, in overweight patients suffering from NAFLD. In this prospective study, fifty Caucasian overweight patients were randomized into three groups (Groups A-C). A personalized moderately hypocaloric Mediterranean diet was prescribed to all patients included in the A and B groups. In addition to the diet, Group B was administered antioxidant supplementation daily and for the period of six months. Group C did not have any type of treatment. The study proved that the Mediterranean diet alone or in association with the antioxidant complex improved anthropometric parameters, lipid profile and reduced hepatic fat accumulation and liver stiffness. However, Group B patients, in which the diet was associated with antioxidant intake, showed not only a significant improvement in insulin sensitivity, but also a more consistent reduction of anthropometric parameters when compared with Group A patients. Taken together, these results support the benefit of antioxidant supplementation in overweight patients with NAFLD.
\end{abstract}

Keywords: non-alcoholic fatty liver disease; Mediterranean diet; insulin resistance; weight; antioxidant

\section{Introduction}

Non-alcoholic fatty liver disease (NAFLD) is an emerging public health issue, being not only a major cause of liver-related morbidity and mortality worldwide, but also an independent risk factor for cardiovascular diseases [1-3]. It refers to a wide spectrum of liver injuries, ranging from steatosis to non-alcoholic steatohepatitis (NASH), fibrosis, cirrhosis and related complications [4,5]. The Dionysos Nutrition and Liver Study and the Dallas Heart Study have calculated that $25 \%$ of Italian and $30 \%$ of American citizens are affected by NAFLD [6,7].

NAFLD is now considered the hepatic border of metabolic syndrome, supported by a high-calorie dietetic regimen, in the presence of a genetic profile characterized by predisposing polymorphisms $[4,8,9]$. Several pathogenic hypotheses on NAFLD development have been formulated. Both the early "two-hit" model and the more recent "multi parallel hit" hypothesis suggest that fat 
accumulation in the hepatocytes exposes the liver to oxidative stress with reactive oxygen species (ROS) production, inflammation unbalance, cellular necrosis and, finally, fibrosis [10-14].

In particular, several pieces of evidence both in animal models and in humans support a key role of oxidative stress in the progression of NAFLD $[13,15]$. An increase in liver and serum content of oxidation products and a decrease in plasma antioxidant activity has been documented in NAFLD patients $[16,17]$. It has also been demonstrated that ROS production is greater in patients with NASH compared with those with simple steatosis [12,18,19], and further studies showed improvement of NASH after taking antioxidant supplements, indirectly supporting an involvement of oxidative stress in the progression of steatosis [20-22].

High-calorie diet represents an underlying mechanism favoring ROS generation and, consequently, increased requirements of antioxidant enzymes [14], such as glutathione reductase, which increases to limit the oxidative damage, particularly in severely obese individuals [15]. NAFLD is frequently asymptomatic and accidentally discovered by blood liver function tests or instrumental investigations.

NAFLD treatment entails a consistent change in lifestyle habits. Literature data and international guidelines have highlighted the health benefits linked to weight loss and physical exercise [23]. In this way, the Mediterranean diet appears to be a perfect fit for NAFLD patients, due to its efficacy on liver status leading to improvement of insulin sensitivity and lipid profile, but also for being a primary form of prevention for NAFLD-related diseases [24-26].

However, there is currently no consensus regarding the pharmacological treatment of NAFLD. Many drugs have been tested and proposed to prevent or improve NAFLD. The most common approach has probably been the use of classical oral anti-diabetic medications including thiazolidinediones, whose use combines insulin-sensitizing and anti-proliferative effects [27,28], as well as lipid lowering agents [23].

Recently, we have described a series of cases involving overweight patients suffering from NAFLD, for which lifestyle changes associated with the administration of a new antioxidant complex supplement, recently introduced in the Italian market (Bilirel (BIL), Pharmaluce, San Marino, Republic of San Marino), have led to decreased liver fat accumulation and weight reduction [29]. The composition of one pill of BIL is silymarin $120 \mathrm{mg}$, chlorogenic acid $7.5 \mathrm{mg}$, protopine $0.04 \mathrm{mg}$, L-methionine $150 \mathrm{mg}$ and L-glutathione $10 \mathrm{mg}$.

This randomized study aims to evaluate the effects of the BIL complex associated with Mediterranean diet on liver fat accumulation, glucose and lipid metabolism and on anthropometric parameters in NAFLD overweight patients.

\section{Patients and Methods}

In this prospective study, 50 Caucasian overweight patients with body mass index (BMI) greater than $25 \mathrm{~kg} / \mathrm{m}^{2}$, who regularly attended our outpatient Gastroenterology clinic, were consecutively enrolled from June 2015-June 2016. Our study protocol is consistent with the ethical guidelines of the Helsinki 1975 Declaration and was approved by the local Ethical Committee (Comitato etico regionale, Sezione Area Centro, Catanzaro, Italy). Informed Consent was obtained for each patient enrolled in the study. Patients affected by hepatitis B and C, with cardiac, renal, autoimmune and metabolic diseases were excluded from the study. Exclusion criteria were also insulin treatment, smoking habits, alcohol intake ( $>20 \mathrm{~g} /$ day), recreational drug use and exposure to environmental toxins known to induce liver steatosis.

Patients were randomized by a systematic sampling procedure into three groups (Groups A-C). A personalized moderately low-calorie Mediterranean diet (1400-1600 kcal/day) was conceived of and prescribed to Group A and B patients for six months. Diet composition was designed to get an animal to vegetable protein ratio of 1:1. The Italian Recommended Dietary Allowances (RDAs) were incorporated to ensure proper vitamin and mineral intake $[30,31]$. The proposed food plan included carbohydrates $(50-60 \%)$, proteins $(15-20 \%$, about $50 \%$ of which were vegetable proteins), mono- and poly-unsaturated fats (less than $30 \%$ ), saturated fat (less than $10 \%$ ), cholesterol (less than $300 \mathrm{mg}$ /day) and fibers (25-30 g/day). 
In association with the diet, Group B patients were administered two pills of BIL complex daily, for six months. This dose of BIL complex is consistent with our previously published data and it is currently used in clinical practice [29]. Daily physical activity was strongly recommended for patients of both Groups A and B [15,32]. Strict observation of dietary and physical activity prescriptions was monitored through a monthly phone interview. Finally, patients of the control group (Group C), who had not undertaken any pharmacological treatment and lifestyle changes, were monitored for the same period, with the medical indication to reduce body weight.

\subsection{Clinical Parameters and Blood Sample Collection}

Anthropometric parameters (body weight, height, waist and hip circumferences), systolic and diastolic blood pressure were assessed according to standard methods. BMI was calculated as body weight divided by height squared $\left(\mathrm{kg} / \mathrm{m}^{2}\right)$. Blood was collected, after $12-14 \mathrm{~h}$ from fasting, by an antecubital venous puncture. Serum or plasma samples were obtained by centrifugation. Serum levels of total cholesterol, low density cholesterol (LDL-C), triglycerides (TG), fasting glucose, insulin, creatinine, urea and standard liver tests, including total bilirubin, aspartate aminotransferase (AST), alanine aminotransferase (ALT) and gamma-glutamyl transpeptidase $(\gamma \mathrm{GT})$, were directly measured using standard automated laboratory methods on Cobas 6000 (Roche, Rotkreuz, Switzerland), by using the relative kits, according to the manufacturer's instructions. To evaluate insulin resistance, we calculated two surrogate indexes: the homeostasis model assessment of insulin resistance (HOMA-IR) and the product of the plasma triglyceride and glucose concentrations (TyG) index [33,34].

\subsection{Steatosis Evaluation}

To diagnose NAFLD, liver ultrasound (US) examination was performed. The hepatic fat accumulation grade was calculated as follows: absent (score 0), mild (score 1), moderate (score 2) and severe (score 3), according to the Hamaguchi score, which uses a 6-point scoring system based on hepatorenal echo contrast, liver brightness, deep attenuation and vascular blurring. This scoring system showed $100 \%$ specificity and $91.7 \%$ sensitivity when compared with liver biopsy [35].

In line with the recent development of new non-invasive methods to detect the presence of liver steatosis, we used also the fatty liver (FL) index, a validated algorithm based on BMI, waist circumference, TG and $\gamma$ GT values, which ranges from $0-100$, with an accuracy of 0.84 (95\% confidence interval (CI) 0.81-0.87) [36]. The FL index is thus calculated as:

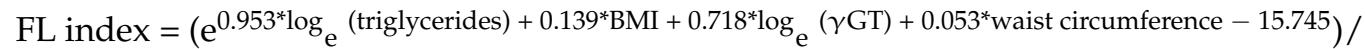

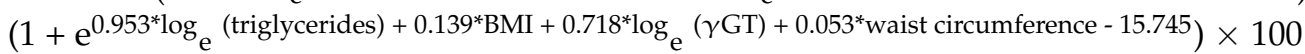

An FL index $<30$ (negative likelihood ratio $=0.2$ ) rules out steatosis, whereas an FL index $\geq 60$ (positive likelihood ratio $=4.3$ ) identifies steatosis.

\subsection{Liver Fibrosis Assessment}

Each patient underwent liver stiffness measurement by transient elastography (TE) (FibroScan ${ }^{\circledR}$, Echosens, Paris, France) using the M probe. This medical device provides a quantifiable estimate of liver stiffness in kilopascal $(\mathrm{kPa})$; measurements were considered representative only if they scored at least 10 valid acquisitions with a success rate $>60 \%$ [37,38]. All measurements were taken by the same operator (experience $>10,000$ measurements) who was unaware of other patient's parameters.

\section{Data Analysis}

Statistical analyses were performed by SPSS 20.0 software for Windows. Each analyte was tested for normality using the Kolmogorov-Smirnov test. Because of the non-normal distribution of the studied variables, they were indicated as the median and interquartile range (IQR). 
The non-parametric Wilcoxon test was used to evaluate the intragroup differences between the anthropometric variables and laboratory parameters at zero time and after six months. To evaluate the intergroup percentage changes in all traits during the time of the study, the non-parametric Mann-Whitney test was performed. The percentage variations from baseline to six months were calculated as $\Delta \mathrm{T} 0 / \mathrm{T} 6=(\mathrm{T} 6-\mathrm{T} 0) / \mathrm{T} 0 \times 100 . \mathrm{A} p$-value less than 0.05 was regarded as statistically significant.

\section{Results}

Clinical, anthropometric and laboratory parameters of all patients stratified in the three groups are reported in Table 1. Patients were subdivided using a systematic random sampling procedure: Group A included 20 patients (120 and 8\%; median age 52 (IQR: 40-60) years); Group B included 20 patients (16 $0^{\top}$ and 4\%; median age 46 (IQR: 40-57) years); Group C included 10 patients (6 $60^{\top}$ and 4\%; median age 33 (IQR: 28-53) years). We did not report side effects or dropouts during the study, and full adherence to the study protocol was registered. Compliance with the study protocol was evaluated through a weekly phone interview for Group A and B patients.

Table 1. Characteristics of the study population stratified into the three groups, at baseline.

\begin{tabular}{|c|c|c|c|}
\hline & Group A $(n=20)$ & Group B $(n=20)$ & Group C $(n=10)$ \\
\hline Age (years) & $52(40-60)$ & $46(40-57)$ & $33(28-43)$ \\
\hline Weight (kg) & $83(80-88)$ & $90(81-92)$ & $84(75-95)$ \\
\hline BMI $\left(\mathrm{kg} / \mathrm{m}^{2}\right)$ & $31(29-33)$ & $29(28-32)$ & $29(27-31)$ \\
\hline Waist circumference $(\mathrm{cm})$ & $108(99-114)$ & $104(100-105)$ & $102(97-110)$ \\
\hline Hip circumference $(\mathrm{cm})$ & $105(102-116)$ & $105(102-110)$ & $105(99-112)$ \\
\hline Systolic blood pressure (mmHg) & $125(120-140)$ & $130(120-140)$ & $120(110-130)$ \\
\hline Diastolic blood pressure (mmHg) & $80(70-90)$ & $80(70-90)$ & $80(60-82)$ \\
\hline $\operatorname{AST}(\mathrm{U} / \mathrm{L})$ & $22(20-25)$ & $22(20-25)$ & $25(23-35)$ \\
\hline $\operatorname{ALT}(\mathrm{U} / \mathrm{L})$ & $22(15-30)$ & $25(21-40)$ & $35(24-58)$ \\
\hline$\gamma \mathrm{GT}(\mathrm{U} / \mathrm{L})$ & $20(16-28)$ & $21(14-31)$ & $21(16-37)$ \\
\hline Total bilirubin (mg/dL) & $0.45(0.38-0.80)$ & $0.40(0.30-0.60)$ & $0.80(0.77-0.90)$ \\
\hline Fasting glucose (mg/dL) & $96(94-110)$ & $101(90-113)$ & $100(95-110)$ \\
\hline Insulin $(\mathrm{mU} / \mathrm{L})$ & $8(4-16)$ & $15(13-21)$ & $7(5-11)$ \\
\hline Triglycerides (mg/dL) & $140(129-157)$ & $101(84-106)$ & $138(115-173)$ \\
\hline Total cholesterol (mg/dL) & $189(178-206)$ & $198(171-213)$ & $176(147-191)$ \\
\hline LDL-C $(\mathrm{mg} / \mathrm{dL})$ & $124(105-134)$ & $122(97-133)$ & $115(99-125)$ \\
\hline Creatinine $(\mathrm{mg} / \mathrm{dL})$ & $0.8(0.7-0.8)$ & $0.8(0.7-0.9)$ & $0.80(0.70-0.90)$ \\
\hline Urea $(\mathrm{mg} / \mathrm{dL})$ & $38(31-43)$ & $38(28-40)$ & $32(26-40)$ \\
\hline HOMA-IR & $2(1-2)$ & $4(3-6)$ & $2(1-3)$ \\
\hline TyG index & $4.7(4.7-4.8)$ & $4.6(4.5-4.8)$ & $4.7(4.7-4.8)$ \\
\hline FL index & $71(56-85)$ & $58(42-69)$ & $67(63-80)$ \\
\hline TE & $8.1(6.7-9.2)$ & $6.9(6.7-7.2)$ & $7.2(5.3-10.1)$ \\
\hline US score & $2(2-3)$ & $2(2-2)$ & $1(0.75-2)$ \\
\hline
\end{tabular}

Continuous variables are expressed as median and quartiles (IQR). BMI: body mass index; AST: aspartate aminotransferase; ALT: alanine aminotransferase; $\gamma$ GT: $\gamma$-glutamyl transpeptidase; LDL: low-density lipoprotein cholesterol; HOMA-IR: homeostasis model assessment-insulin resistance; TyG index: triglyceride-glucose index; FL index: fatty liver index; TE: transient elastography; US score: ultrasound score.

Group A patients showed after six months a statistically-significant decrease in weight, BMI, waist and hip circumference $(p=0.0001)$ and an improvement of $\gamma \mathrm{GT}(p=0.024)$, lipid profile with a significant decrease in TG $(p=0.0001)$, total cholesterol $(p=0.0001)$ and LDL-C $(p=0.005)$. A significant decrease was also detected in the FL index $(p=0.002)$ and TE values $(p=0.0001)$ (Table 2$)$. 
Table 2. Comparison of anthropometric, biochemical and clinical features at baseline (T0) and after six months (T6) of treatment in patients following a low-calorie diet (Group A).

\begin{tabular}{cccc}
\hline & \multicolumn{2}{c}{ Group A } & \\
\hline & T0 & T6 & $p$ \\
\hline Weight $(\mathrm{kg})$ & $83(80-88)$ & $78(75-80)$ & 0.0001 \\
BMI $\left(\mathrm{kg} / \mathrm{m}^{2}\right)$ & $31(29-33)$ & $29(27-31)$ & 0.0001 \\
Waist circumference (cm) & $108(99-114)$ & $102(98-111)$ & 0.0001 \\
Hip circumference (cm) & $105(102-116)$ & $102(99-110)$ & 0.0001 \\
Systolic blood pressure (mmHg) & $125(120-140)$ & $125(120-130)$ & 0.121 \\
Diastolic blood pressure (mmHg) & $80(70-90)$ & $80(70-80)$ & 0.755 \\
AST (U/L) & $22(20-25)$ & $23(21-25)$ & 0.101 \\
ALT (U/L) & $22(15-30)$ & $25(18-31)$ & 0.497 \\
$\gamma$ GT (U/L) & $20(16-28)$ & $25(21-31)$ & 0.024 \\
Total bilirubin (mg/dL) & $0.45(0.38-0.80)$ & $0.45(0.30-0.80)$ & 0.436 \\
Fasting glucose (mg/dL) & $96(94-110)$ & $90(88-102)$ & 0.258 \\
Insulin (mU/L) & $8(4-16)$ & $7(3-15)$ & 0.777 \\
Triglycerides (mg/dL) & $140(129-157)$ & $85(75-135)$ & 0.0001 \\
Total cholesterol (mg/dL) & $189(178-206)$ & $156(143-185)$ & 0.0001 \\
LDL-C (mg/dL) & $124(105-134)$ & $102(92-115)$ & 0.005 \\
Creatinine (mg/dL) & $0.8(0.7-0.8)$ & $0.8(0.7-0.9)$ & 0.218 \\
Urea (mg/dL) & $38(31-43)$ & $30(27-34)$ & 0.007 \\
HOMA-IR & $1.9(0.9-2.4)$ & $1.8(0.6-3.4)$ & 0.985 \\
TyG index & $4.7(4.7-4.8)$ & $4.5(4.4-4.8)$ & 0.100 \\
FL index & $71(56-85)$ & $45(39-69)$ & 0.002 \\
TE & $8.1(6.7-9.2)$ & $6.0(5.1-7.0)$ & 0.0001 \\
US score & $2(2-3)$ & $2(1-2)$ & 0.0001 \\
\hline
\end{tabular}

Non-parametric Wilcoxon test. A $p$-value less than 0.05 is considered statistically significant. BMI: body mass index; AST: aspartate aminotransferase; ALT: alanine aminotransferase; $\gamma \mathrm{GT}: \gamma$-glutamyl transpeptidase; LDL: low-density lipoprotein cholesterol; HOMA-IR: homeostasis model assessment-insulin resistance; TyG index: triglyceride-glucose index; FL index: fatty liver index; TE: transient elastography; US score: ultrasound score.

In Group B patients, a statistically-significant decrease after six months of treatment was observed in body weight $(p=0.02)$, BMI $(p=0.0001)$, waist $(p=0.0001)$ and hip circumference $(p=0.001)$, systolic blood pressure $(p=0.012)$, as well as in ALT $(p=0.007)$, fasting glucose $(p=0.007)$, insulin $(p=0.0001)$, TG $(p=0.011)$, total cholesterol $(p=0.0001)$ and LDL-C $(p=0.016)$ blood levels.

Moreover, in Group B, we observed not only a significant decrease in the FL index $(p=0.003)$ and TE values $(p=0.0001)$, but also in both insulin resistance indexes, HOMA-IR $(p=0.001)$ and TyG $(p=0.005)$ (Table 3). Conversely, in the control group $(C)$, statistically-significant improvements in AST $(p=0.023), \gamma \mathrm{GT}(p=0.036)$, insulin $(p=0.041)$, total cholesterol $(p=0.025)$ and LDL-C $(p=0.028)$ blood levels were reported after six months (Table 4). Group C patients even showed an increase in the HOMA-IR index $(p=0.024)$.

Table 3. Comparison of anthropometric, biochemical and clinical features at baseline (T0) and after six months (T6) of treatment in patients following a low-calorie diet in association with the Bilirel (BIL) complex (Group B).

\begin{tabular}{cccc}
\hline & \multicolumn{3}{c}{ Group B } \\
\hline & T0 & T6 & $p$ \\
\hline Weight $(\mathrm{kg})$ & $90(81-92)$ & $81(74-86)$ & 0.002 \\
BMI $\left(\mathrm{kg} / \mathrm{m}^{2}\right)$ & $29(28-32)$ & $27(25-28)$ & 0.0001 \\
Waist circumference $(\mathrm{cm})$ & $104(100-105)$ & $98(96-100)$ & 0.0001 \\
Hip circumference $(\mathrm{cm})$ & $105(102-110)$ & $101(99-102)$ & 0.001 \\
Diastolic blood pressure $(\mathrm{mmHg})$ & $80(70-90)$ & $80(70-80)$ & 0.285 \\
Systolic blood pressure $(\mathrm{mmHg})$ & $130(120-140)$ & $120(120-130)$ & 0.012 \\
\hline
\end{tabular}


Table 3. Cont.

\begin{tabular}{cccc}
\hline & \multicolumn{3}{c}{ Group B } \\
\hline & T0 & T6 & $p$ \\
\hline AST (U/L) & $22(20-25)$ & $21(18-32)$ & 0.955 \\
ALT (U/L) & $25(21-40)$ & $25(17-25)$ & 0.007 \\
$\gamma$ GT (U/L) & $21(14-31)$ & $24(16-30)$ & 0.175 \\
Total bilirubin (mg/dL) & $0.40(0.30-0.60)$ & $0.50(0.40-0.60)$ & 0.084 \\
Fasting glucose (mg/dL) & $101(90-113)$ & $93(85-96)$ & 0.007 \\
Insulin (mU/L) & $15(13-21)$ & $9(7-10)$ & 0.0001 \\
Triglycerides (mg/dL) & $106(100-139)$ & $75(61-92)$ & 0.011 \\
Total cholesterol (mg/dL) & $198(171-213)$ & $152(140-180)$ & 0.0001 \\
LDL-C (mg/dL) & $122(97-133)$ & $98(78-120)$ & 0.016 \\
Creatinine (mg/dL) & $0.8(0.7-0.9)$ & $95(78-120)$ & 0.409 \\
Urea (mg/dL) & $38(28-40)$ & $39(28-44)$ & 0.497 \\
HOMA-IR & $4(3-6)$ & $2(1-2)$ & 0.001 \\
TyG index & $4.6(4.5-4.8)$ & $4.5(4.5-4.7)$ & 0.005 \\
FL index & $58(42-69)$ & $38(29-45)$ & 0.003 \\
TE & $6.9(6.7-7.2)$ & $5.0(4.7-5.2)$ & 0.0001 \\
US score & $2(2-2)$ & $0(0-1)$ & 0.0001 \\
\hline
\end{tabular}

Non-parametric Wilcoxon test. A $p$-value less than 0.05 would be considered statistically significant. BMI: body mass index; AST: aspartate aminotransferase; ALT: alanine aminotransferase; $\gamma \mathrm{GT}$ : $\gamma$-glutamyl transpeptidase; LDL: low-density lipoprotein cholesterol; HOMA-IR: homeostasis model assessment-insulin resistance; TyG index: triglyceride-glucose index; FL index: fatty liver index; TE: transient elastography; US score: ultrasound score.

Table 4. Comparison of anthropometric, biochemical and clinical features at baseline (T0) and after six months (T6) in control patients (Group C).

\begin{tabular}{|c|c|c|c|}
\hline & \multicolumn{2}{|c|}{ Group C } & \multirow[b]{2}{*}{$p$} \\
\hline & T0 & T6 & \\
\hline Weight (kg) & $84(75-95)$ & 85 (75-95) & 0.214 \\
\hline BMI $\left(\mathrm{kg} / \mathrm{m}^{2}\right)$ & $29(27-31)$ & $29(27-30)$ & 0.223 \\
\hline Waist circumference $(\mathrm{cm})$ & $102(97-110)$ & $102(99-111)$ & 0.334 \\
\hline Hip circumference $(\mathrm{cm})$ & $105(99-112)$ & $106(99-113)$ & 0.389 \\
\hline Systolic Blood Pressure (mmHg) & $120(110-130)$ & $120(120-132)$ & 0.066 \\
\hline Diastolic Blood Pressure (mmHg) & $80(60-82)$ & $80(70-82)$ & 0.102 \\
\hline AST $(\mathrm{U} / \mathrm{L})$ & $25(23-35)$ & $29(24-56)$ & 0.023 \\
\hline $\operatorname{ALT}(\mathrm{U} / \mathrm{L})$ & $35(24-58)$ & $40(32-52)$ & 0.878 \\
\hline$\gamma \mathrm{GT}(\mathrm{U} / \mathrm{L})$ & $21(16-37)$ & $29(21-37)$ & 0.036 \\
\hline Total Bilirubin (mg/dL) & $0.80(0.77-0.90)$ & $0.80(0.75-0.90)$ & 0.257 \\
\hline Fasting Glucose (mg/dL) & $100(95-110)$ & $101(96-110)$ & 0.395 \\
\hline Insulin $(\mathrm{mU} / \mathrm{L})$ & $7(5-11)$ & $9(6-16)$ & 0.041 \\
\hline Triglycerides (mg/dL) & $138(115-173)$ & $143(138-173)$ & 0.241 \\
\hline Total Cholesterol (mg/dL) & $176(147-191)$ & $152(140-180)$ & 0.025 \\
\hline LDL-C $(\mathrm{mg} / \mathrm{dL})$ & $115(99-125)$ & $78(54-95)$ & 0.028 \\
\hline Creatinine $(\mathrm{mg} / \mathrm{dL})$ & $0.80(0.70-0.90)$ & $0.8(0.77-0.90)$ & 0.083 \\
\hline Urea $(\mathrm{mg} / \mathrm{dL})$ & $32(26-40)$ & $31(28-36)$ & 0.776 \\
\hline HOMA-IR & $1.8(1.2-2.8)$ & $2.3(1.6-3.9)$ & 0.024 \\
\hline TyG index & $4.7(4.7-4.8)$ & $4.8(4.8-4.9)$ & 0.132 \\
\hline FL index & $67(63-80)$ & $69(68-83)$ & 0.066 \\
\hline Fibroscan & $7.2(5.3-10.1)$ & $8.5(6.3-9.7)$ & 0.683 \\
\hline US score & $1(0.75-2)$ & $1(1-2)$ & 0.705 \\
\hline
\end{tabular}

Non-parametric Wilcoxon test. A $p$-value less than 0.05 is considered statistically significant. BMI: body mass index; AST: aspartate aminotransferase; ALT: alanine aminotransferase; $\gamma$ GT: $\gamma$-glutamyl transpeptidase; LDL: low-density lipoprotein cholesterol; HOMA-IR: homeostasis model assessment-insulin resistance; TyG index: triglyceride-glucose index; FL index: fatty liver index; TE: transient elastography; US score: ultrasound score.

Intergroup percentage changes in all traits $(\Delta \mathrm{T} 0 / \mathrm{T} 6)$ from baseline (T0) to six months (T6) were evaluated by the Mann-Whitney test (Table 5). The analysis between Groups A and C showed 
statistically-significant variations in anthropometric parameters, such as weight and BMI $(p=0.0001)$, waist $(p=0.0001)$, hip circumference $(p=0.001)$, lipid profile: TG $(p=0.001)$ and total cholesterol $(p=0.0001)$, insulin sensitivity: insulin $(p=0.045)$, HOMA-IR $(p=0.021)$, TyG index $(p=0.020)$, but also in the FL index $(p=0.017)$ and TE values $(p=0.001)$.

Table 5. Effects of diet and diet/BIL complex association on anthropometric, clinical and biochemical parameters.

\begin{tabular}{|c|c|c|c|c|}
\hline & Group A & Group B & Group C & $p$ \\
\hline Weight (kg) & $6 \%(-)$ & $7 \%(-)$ & $0.5 \%(-)$ & $\begin{array}{c}\text { A vs. C } \mathbf{0 . 0 0 0 1} \\
\text { B vs. C } \mathbf{0 . 0 3 0} \\
\text { A vs. B } 0.665\end{array}$ \\
\hline BMI $\left(\mathrm{kg} / \mathrm{m}^{2}\right)$ & $7.5 \%(-)$ & $9 \%(-)$ & $0.45 \%(-)$ & $\begin{array}{c}\text { A vs. C } \mathbf{0 . 0 0 0 1} \\
\text { B vs. C } \mathbf{0 . 0 0 0 1} \\
\text { A vs. B } 0.935\end{array}$ \\
\hline Waist circumference $(\mathrm{cm})$ & $2.8 \%(-)$ & $6 \%(-)$ & $0.3 \%(-)$ & $\begin{array}{c}\text { A vs. C } \mathbf{0 . 0 0 0 1} \\
B \text { vs. C } \mathbf{0 . 0 0 0 1} \\
\text { A vs. B } 0.030\end{array}$ \\
\hline Hip circumference $(\mathrm{cm})$ & $3.3 \%(-)$ & $4 \%(-)$ & $0.7 \%(-)$ & $\begin{array}{l}\text { A vs. C } \mathbf{0 . 0 0 1} \\
\text { B vs. C } \mathbf{0 . 0 0 1} \\
\text { A vs. B } 0.206\end{array}$ \\
\hline Fasting glucose (mg/dL) & $3.5 \%(-)$ & $11 \%(-)$ & $0.5 \%(-)$ & $\begin{array}{l}\text { A vs. C } 0.724 \\
\text { B vs. C } \mathbf{0 . 0 0 6} \\
\text { A vs. B } \mathbf{0 . 0 1 6}\end{array}$ \\
\hline Insulin (mU/L) & $10 \%(+)$ & $38 \%(-)$ & $25 \%(+)$ & $\begin{array}{l}\text { A vs. C } 0.045 \\
\text { B vs. C } \mathbf{0 . 0 0 0 1} \\
\text { A vs. B } \mathbf{0 . 0 0 0 1}\end{array}$ \\
\hline Triglycerides (mg/dL) & $32.16 \%(-)$ & $21 \%(-)$ & $2.8 \%(+)$ & $\begin{array}{l}\text { A vs. C } \mathbf{0 . 0 0 1} \\
\text { B vs. C } \mathbf{0 . 0 0 2} \\
\text { A vs. B } 0.935\end{array}$ \\
\hline Total cholesterol (mg/dL) & $14.8 \%(-)$ & $17 \%(-)$ & $9.3 \%(+)$ & $\begin{array}{c}\text { A vs. C } \mathbf{0 . 0 0 0 1} \\
\text { B vs. C } \mathbf{0 . 0 0 0 1} \\
\text { A vs. B } 0.626\end{array}$ \\
\hline LDL-C (mg/dL) & $15 \%(-)$ & $9 \%(-)$ & $29 \%(-)$ & $\begin{array}{l}\text { A vs. C } 0.217 \\
\text { B vs. C } 0.234 \\
\text { A vs. B } 0.705\end{array}$ \\
\hline HOMA-IR & $6.2 \%(+)$ & $43 \%(-)$ & $46 \%(+)$ & $\begin{array}{c}\text { A vs. C } 0.021 \\
\text { B vs. C } \mathbf{0 . 0 0 1} \\
\text { A vs. B } \mathbf{0 . 0 0 0 1}\end{array}$ \\
\hline TyG index & $3.3 \%(-)$ & $1.2 \%(-)$ & $1 \%(+)$ & $\begin{array}{l}\text { A vs. C } \mathbf{0 . 0 2 0} \\
\text { B vs. C } \mathbf{0 . 0 1 0} \\
\text { A vs. B } 0.131\end{array}$ \\
\hline FL index & $19 \%(-)$ & $27 \%(-)$ & $4.7 \%(+)$ & $\begin{array}{c}\text { A vs. C } \mathbf{0 . 0 1 7} \\
\text { B vs. C } \mathbf{0 . 0 0 0 1} \\
\text { A vs. B } 0.626\end{array}$ \\
\hline $\mathrm{TE}$ & $21 \%(-)$ & $27 \%(-)$ & $8.7 \%(+)$ & $\begin{array}{c}\text { A vs. C } \mathbf{0 . 0 0 1} \\
\text { B vs. C } \mathbf{0 . 0 0 0 1} \\
\text { A vs. B } 0.053\end{array}$ \\
\hline
\end{tabular}

The non-parametric Mann-Whitney test was used to evaluate the intergroup percentage changes $(p<0.05)$; (-) reduction, (+) increase. BMI: body mass index; LDL: low-density lipoprotein cholesterol; HOMA-IR: homeostasis model assessment-insulin resistance; TyG index: triglyceride-glucose index; FL index: fatty liver index; TE: transient elastography.

A comparison between Groups B and C showed substantially the same differences in anthropometric parameters described above (weight $(p=0.030)$, BMI $(p=0.0001)$, waist $(p=0.0001)$ and 
hip circumference $(p=0.001)$ ). A significant improvement in glycemic metabolism (insulin $(p=0.0001)$, fasting glucose levels $(p=0.006)$, HOMA-IR $(p=0.001)$, TyG index $(p=0.01))$, in steatosis evaluation by the FL index $(p=0.0001)$ and TE values $(p=0.0001)$ has also been observed.

However, Group B patients, who underwent both diet and BIL complex treatment, showed a statistically-significant reduction in fasting glucose $(p=0.016)$, insulin levels $(p=0.0001)$ and, consequently, HOMA-IR index ( $p=0.0001)$, as compared to Group A patients (Figure 1). Finally, inter-group analysis of all tested parameters showed that fasting glucose and insulin level, HOMA-IR, FL index and TE values were significantly lower in Group B than in Group A, after the treatment period (Table 5).
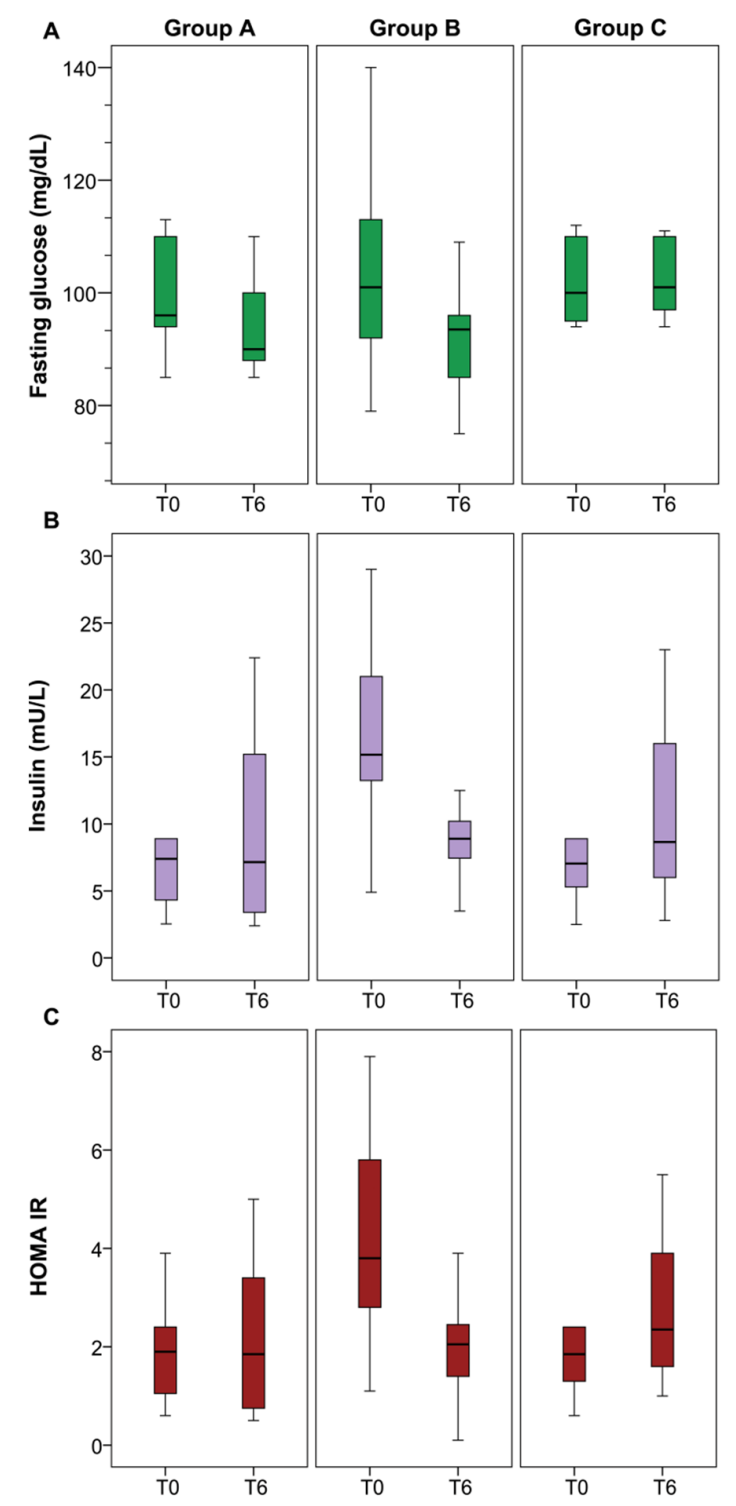

Figure 1. Comparison of fasting glucose (A); insulin (B) and HOMA-IR (C) at baseline (T0) and after six months (T6), among the three groups (Group A = diet; Group B = diet/BIL complex; Group C = control, without diet and antioxidant supplementation).

\section{Discussion}

Despite the rapidly growing recognition of NAFLD over the last few decades, the treatment of this condition remains debated $[39,40]$. In the clinical management of NAFLD patients, a dietary change 
and increased physical exercise are essential to reduce body weight, in order to improve metabolic parameters and normalize the biochemical blood profile, as well as transaminase levels [24]. The "ideal" treatment for NAFLD should reduce the liver damage and its progression by reducing anthropometric parameters, by improving insulin resistance and impairment in glucose and lipid metabolism and by reducing the cytokine-mediated pathophysiological link between adipose tissue and liver [41]. The traditional Mediterranean diet is a dietary pattern that was associated with favorable health impact, in particular on cardiovascular diseases, cancer and in the treatment of metabolic syndromes [42]. Carotenoids, fibers and folic acid, which are basic components of this diet, can play a pivotal role in preventing or slowing down the oxidative stress process. In addition, vegetables, which are the staple foods included in the Mediterranean diet, are the main source of phytosterols, known as natural cholesterol-lowering agents, reducing cardiovascular risk [43,44].

Several pharmaceutical agents are currently being evaluated for the treatment of NAFLD, and NASH in particular. However, no single therapy has been approved so far [23,45]. On this basis, the beneficial effects of complementary medicine, and particularly of herbal extracts, on NAFLD patients have received increasing attention in the last few years. The use of this approach has many advantages, including worldwide availability, minimal reported side effects and wide application due to low treatment costs [46].

However, literature data are often inconclusive on this topic, due to the high number of biases found in many trials and to the limited number of studies testing single herbal remedies [47].

In the last two decades, several studies have emphasized the benefits in the NAFLD treatment of Silybum marianum, commonly called milk thistle (MT), a plant native to the Mediterranean area, which has been used for many centuries to treat liver diseases [48,49]. The active complex of MT is a lipophilic extract from the seeds of the plant, and it is composed by three flavonolignan isomers, silybin, silydianin and silychristin, collectively called silymarin.

Studies of patients with NAFLD showed that silymarin treatment was associated with positive changes in insulin resistance and transaminase serum levels [50,51]. Loguercio et al., in a multicenter phase III double-blind clinical trial, showed that MT extracts, after 12 months, led to an improvement of insulin resistance, liver enzymes and liver histology, without any increase in body weight in NAFLD patients [52]. More recently, in a randomized clinical study, we have found out that Mediterranean diet, in association with silymarin and other antioxidants, is able to induce, after six months, significant changes in glucose and lipid metabolism [53].

According to these data, in our cohort, we demonstrated an improvement of BMI, waist and hip circumference, TG, total cholesterol and LDL-C serum level in all patients who followed the Mediterranean diet for a period of six months (Group A and B). The diet also led to the decrease of intra-hepatic fat accumulation, evaluated by the FL index, and of liver stiffness, assessed by TE. However, in the overweight NAFLD Group B patients, who followed the Mediterranean diet in association with BIL antioxidant treatment, we reported the statistical reduction of the HOMA-IR and the TyG index, two surrogate indexes widely used to evaluate insulin resistance.

The changes in glucose and lipid metabolism described in Group B can be explained also by the presence of chlorogenic acid, one component of the BIL complex. Chlorogenic acid is one of the most abundant polyphenols in the human diet. It is contained in coffee, fruits and vegetables and displays many biological properties, such as antidiabetic effects by stimulating glucose uptake in both insulin-sensitive and insulin-resistant adipocytes and by improving early fasting glucose and insulin responses [54]. The metabolic changes observed in our study can be explained by the synergic action of the Mediterranean diet in association with chlorogenic acid and silymarin.

Another component of the BIL complex is protopine, an isoquinoline alkaloid present in Fumaria officinalis, with antioxidant and choleretic properties that inhibit the production of pro-inflammatory cytokines [55]. Our data suggest that protopine could be a potential candidate for NAFLD treatment. 
The increase in oxidative stress and free radical production observed in NAFLD lead not only to increased consumption of glutathione, the major intra-cellular antioxidant, but it also reduces the activity of s-adenosyl-L-methionine, the main biological methyl donor and a precursor of glutathione, essential for protecting antioxidant pathways [56]. Recent studies suggest that the reduction of glutathione levels, in combination with lower ATP availability due to mitochondrial deregulation, leads to an unbalance of reactive oxygen species production and to the subsequent progression of hepatic injury [57]. In this context, the administration of reduced glutathione and methionine can help to restore the oxidative balance.

The BIL antioxidant complex treatment alone, not in association with physical activity and a calorie-controlled diet, is not effective in improving insulin resistance. However, our data confirm the possible therapeutic role of this antioxidant complex as a complementary approach to the treatment of overweight NAFLD patients and in particular in the management of insulin resistance in NAFLD-related pathologies.

An important goal for modern hepatologists is to find effective non-invasive diagnostic approaches to NAFLD. In the last two decades, non-invasive diagnostic modalities for NAFLD have been investigated. On the basis of literature data, three non-invasive methods have been employed in the present study for the evaluation of NAFLD. In addition to the US examination, in particular, the FL index and TE have been used to assess respectively hepatic fat accumulation and liver stiffness. The FL index is an accurate and easy to employ predictor score to define steatosis presence that utilizes routine measurements in clinical practice such as a BMI, waist circumference, triglycerides and $\gamma \mathrm{GT}$ [36]. In this way, the clinical use of the FL index is useful to identify patients with NAFLD to include in an outpatient lifestyle change program. The data on the reduction of hepatic fat accumulation were also confirmed by the reduction of the Hamaguchi score at the US examination in Groups A and B, compared to Group C.

TE is a non-invasive tool for the evaluation of liver damage that demonstrated good accuracy in quantifying the levels of hepatic stiffness and to define fibrosis, in patients with liver diseases and in particular with NAFLD [37]. This technique is reliable, fast and reproducible, with a good intra- and inter-observer agreement, thus allowing for population-wide screening and disease follow-up.

Finally, our study clearly shows that patients following a balanced diet and taking the antioxidant complex had a more significant attenuation of insulin resistance, hepatic fat accumulation and liver stiffness than patients following the diet alone. These results supported the effectiveness of the BIL complex to reduce liver fatty acid infiltration and its related damages, by positively influencing the mitochondrial function and by reducing oxidative stress.

\section{Conclusions}

Our study confirms that the Mediterranean diet can improve anthropometric parameters and lipid profile and can contribute to reducing hepatic fat accumulation and liver stiffness. Moreover, the association of this dietetic regimen with antioxidant supplementation can contribute to improving the insulin sensitivity parameters. These data support a possible role of antioxidant supplementation as a coadjuvant therapy in patients with NAFLD.

Author Contributions: Ludovico Abenavoli designed the study, performed the research of the literature data and wrote the paper. Marta Greco performed the statistical analysis and wrote the paper. Natasa Milic performed the research of the literature data and critically revised the paper. Francesca Accattato performed the statistical analysis and revised the paper. Daniela Foti critically revised the paper. Elio Gulletta and Francesco Luzza analyzed the data and drafted the article.

Conflicts of Interest: The authors confirm that they have no conflicts of interest whatsoever, not even with the company that produces Bilirel (Pharmaluce, Republic of San Marino). This is in line with our conflicts of interest statement. 


\section{References}

1. Masarone, M.; Federico, A.; Abenavoli, L.; Loguercio, C.; Persico, M. Non alcoholic fatty liver: Epidemiology and natural history. Rev. Recent Clin. Trials 2014, 9, 126-133. [CrossRef] [PubMed]

2. Bellentani, S. The epidemiology of non-alcoholic fatty liver disease. Liver Int. 2017, 37, 81-84. [CrossRef] [PubMed]

3. Lonardo, A.; Ballestri, S.; Guaraldi, G.; Nascimbeni, F.; Romagnoli, D.; Zona, S.; Targher, G. Fatty liver is associated with an increased risk of diabetes and cardiovascular disease-Evidence from three different disease models: NAFLD, HCV and HIV. World J. Gastroenterol. 2016, 22, 9674-9693. [CrossRef] [PubMed]

4. Abenavoli, L.; Milic, N.; Di Renzo, L.; Preveden, T.; Medić-Stojanoska, M.; De Lorenzo, A. Metabolic aspects of adult patients with nonalcoholic fatty liver disease. World J. Gastroenterol. 2016, 22, 7006-7016. [CrossRef] [PubMed]

5. Kobyliak, N.; Abenavoli, L. The role of liver biopsy to assess non-alcoholic fatty liver disease. Rev. Recent Clin. Trials 2014, 9, 159-169. [CrossRef] [PubMed]

6. Bedogni, G.; Miglioli, L.; Masutti, F.; Castiglione, A.; Crocè, L.S.; Tiribelli, C.; Bellentani, S. Incidence and natural course of fatty liver in the general population: The Dionysos study. Hepatology 2007, 46, 1387-1391. [CrossRef] [PubMed]

7. Turer, A.T.; Browning, J.D.; Ayers, C.R.; Das, S.R.; Khera, A.; Vega, G.L.; Grundy, S.M.; Scherer, P.E. Adiponectin as an independent predictor of the presence and degree of hepatic steatosis in the Dallas heart study. J. Clin. Endocrinol. Metab. 2012, 97, E982-E986. [CrossRef] [PubMed]

8. Romeo, S.; Kozlitina, J.; Xing, C.; Pertsemlidis, A.; Cox, D.; Pennacchio, L.A.; Boerwinkle, E.; Cohen, J.C.; Hobbs, H.H. Genetic variation in PNPLA3 confers susceptibility to nonalcoholic fatty liver disease. Nat. Genet. 2008, 40, 1461-1465. [CrossRef] [PubMed]

9. Chiefari, E.; Tanyolaç, S.; Iiritano, S.; Sciacqua, A.; Capula, C.; Arcidiacono, B.; Nocera, A.; Possidente, K.; Baudi, F.; Ventura, V.; et al. A polymorphism of HMGA1 is associated with increased risk of metabolic syndrome and related components. Sci. Rep. 2013, 3, 1491. [CrossRef] [PubMed]

10. Day, C.P.; James, O.F. Steatohepatitis: A tale of two "hits"? Gastroenterology 1998, 114, 842-845. [CrossRef]

11. Tilg, H.; Moschen, A.R. Evolution of inflammation in nonalcoholic fatty liver disease: The multiple parallel hits hypothesis. Hepatology 2010, 52, 1836-1846. [CrossRef] [PubMed]

12. Day, C.P. Pathogenesis of steatohepatitis. Best Pract. Res. Clin. Gastroenterol. 2002, 16, 663-678. [CrossRef] [PubMed]

13. Gambino, R.; Musso, G.; Cassader, M. Redox balance in the pathogenesis of nonalcoholic fatty liver disease: Mechanisms and therapeutic opportunities. Antioxid. Redox Signal. 2011, 15, 1325-1365. [CrossRef] [PubMed]

14. Beltowsky, J.; Wójcicka, G.; Górny, D.; Marciniak, A. The effect of dietary-induced obesity on lipid peroxidation, antioxidant enzymes and total plasma antioxidant capacity. Physiol. Pharmacol. 2000, 51, 883-896.

15. Accattato, F.; Greco, M.; Pullano, S.A.; Carè, I.; Fiorillo, A.S.; Pujia, A.; Montalcini, T.; Foti, D.P.; Brunetti, A.; Gulletta, E. Effects of acute physical exercise on oxidative stress and inflammatory status in young, sedentary obese subjects. PLoS ONE 2017, 12, e0178900. [CrossRef] [PubMed]

16. Loguercio, C.; De Simone, T.; D’Auria, M.V.; de Sio, I.; Federico, A.; Tuccillo, C.; Abbatecola, A.M.; Del Vecchio Blanco, C.; Italian AISF Clinical Group. Non-alcoholic fatty liver disease: A multicentre clinical study by the Italian association for the study of the liver. Dig. Liver Dis. 2004, 36, 398-405. [CrossRef]

17. Garcia-Monzon, C.; Martin-Perez, E.; Iacono, O.L.; Fernández-Bermejo, M.; Majano, P.L.; Apolinario, A.; Larrañaga, E.; Moreno-Otero, R. Characterization of pathogenic and prognostic factors of non-alcoholic steatohepatitis associated with obesity. J. Hepatol. 2000, 33, 716-724. [CrossRef]

18. Seki, S.; Kitada, T.; Yamada, T.; Sakaguchi, H.; Nakatani, K.; Wakasa, K. In situ detection of lipid peroxidation and oxidative DNA damage in non-alcoholic fatty liver disease. J. Hepatol. 2002, 37, 56-62. [CrossRef]

19. Chalasani, N.; Deeg, M.A.; Crabb, D.W. Systemic levels of lipid peroxidation and its metabolic and dietary correlates in patients with non-alcoholic steatohepatitis. Am. J. Gastroenterol. 2004, 99, 1497-1502. [CrossRef] [PubMed]

20. Merat, S.; Malekzadeh, R.; Sohrabi, M.R.; Sotoudeh, M.; Rakhshani, N.; Sohrabpour, A.A.; Naserimoghadam, S. Probucol in the treatment of non-alcoholic steatohepatitis: A double-blind randomized controlled study. J. Hepatol. 2003, 38, 414-418. [CrossRef] 
21. Harrison, S.A.; Torgerson, S.; Hayashi, P.; Ward, J.; Schenker, S. Vitamin E and vitamin C treatment improves fibrosis in patients with non-alcoholic steatohepatitis. Am. J. Gastroenterol. 2003, 98, 2485-2490. [CrossRef] [PubMed]

22. Musso, G.; Gambino, R.; De Michieli, F.; Cassader, M.; Rizzetto, M.; Durazzo, M.; Fagà, E.; Silli, B.; Pagano, G. Dietary habits and their relations to insulin resistance and postprandial lipemia in nonalcoholic steatohepatitis. Hepatology 2003, 37, 909-916. [CrossRef] [PubMed]

23. European Association for the Study of the Liver (EASL); European Association for the Study of Diabetes (EASD); European Association for the Study of Obesity (EASO). EASL-EASD-EASO clinical practice guidelines for the management of non-alcoholic fatty liver disease. J. Hepatol. 2016, 64, 1388-1402. [CrossRef]

24. Abenavoli, L.; Milic, N.; Peta, V.; Alfieri, F.; De Lorenzo, A.; Bellentani, S. Alimentary regimen in non-alcoholic fatty liver disease: Mediterranean diet. World J. Gastroenterol. 2014, 20, 16831-16840. [CrossRef] [PubMed]

25. Godos, J.; Federico, A.; Dallio, M.; Scazzina, F. Mediterranean diet and nonalcoholic fatty liver disease: Molecular mechanisms of protection. Int. J. Food Sci. Nutr. 2017, 68, 18-27. [CrossRef] [PubMed]

26. Abellán Alemán, J.; Zafrilla Rentero, M.P.; Montoro-García, S.; Mulero, J.; Pérez Garrido, A.; Leal, M.; Guerrero, L.; Ramos, E.; Ruilope, L.M. Adherence to the "Mediterranean diet" in Spain and its relationship with cardiovascular risk (DIMERICA study). Nutrients 2016, 8, 680. [CrossRef] [PubMed]

27. Chang, E.; Park, C.Y.; Park, S.W. Role of thiazolidinediones, insulin sensitizers, in non-alcoholic fatty liver disease. J. Diabetes Investig. 2013, 4, 517-524. [CrossRef] [PubMed]

28. Costa, V.; Foti, D.; Paonessa, F.; Chiefari, E.; Palaia, L.; Brunetti, G.; Gulletta, E.; Fusco, A.; Brunetti, A. The insulin receptor: A new anticancer target for peroxisome proliferator-activated receptor-gamma (PPARgamma) and thiazolidinedione-PPARgamma agonists. Endocr-Relat. Cancer 2008, 15, 325-335. [CrossRef] [PubMed]

29. Abenavoli, L.; Peta, V.; Milic, N. Lifestyle changes associated with a new antioxidant formulation in non-alcoholic fatty liver disease: A case series. Ann. Hepatol. 2015, 14, 121-126. [PubMed]

30. De Lorenzo, A.; Noce, A.; Bigioni, M.; Calabrese, V.; Della Rocca, D.G.; Di Daniele, N.; Tozzo, C.; Di Renzo, L. The effects of Italian Mediterranean organic diet (IMOD) on health status. Curr. Pharm. Des. 2010, 16, 814-824. [CrossRef] [PubMed]

31. Greco, M.; Chiefari, E.; Montalcini, T.; Accattato, F.; Costanzo, F.S.; Pujia, A.; Foti, D.; Brunetti, A.; Gulletta, E. Early effects of a hypocaloric, Mediterranean diet on laboratory parameters in obese individuals. Mediat. Inflamm. 2014, 2014, 750860. [CrossRef] [PubMed]

32. Keating, S.E.; George, J.; Johnson, N.A. The benefits of exercise for patients with non-alcoholic fatty liver disease. Expert Rev. Gastroenterol. Hepatol. 2015, 9, 1247-1250. [CrossRef] [PubMed]

33. Karne, R.J.; Chen, H.; Quon, M.J. Diagnosing insulin resistance by simple quantitative methods in subjects with normal glucose metabolism. Diabetes Care 2004, 27, 1247-1248. [CrossRef] [PubMed]

34. Simental-Mendía, L.E.; Rodríguez-Morán, M.; Guerrero-Romero, F. The product of fasting glucose and triglycerides as surrogate for identifying insulin resistance in apparently healthy subjects. Metab. Syndr. Relat. Disord. 2008, 6, 299-304. [CrossRef] [PubMed]

35. Hamaguchi, M.; Kojima, T.; Itoh, Y.; Harano, Y.; Fujii, K.; Nakajima, T.; Kato, T.; Takeda, N.; Okudam, J.; Ida, K.; et al. The severity of ultrasonographic findings in nonalcoholic fatty liver disease reflects the metabolic syndrome and visceral fat accumulation. Am. J. Gastroenterol. 2007, 102, 2708-2715. [CrossRef] [PubMed]

36. Bedogni, G.; Bellentani, S.; Miglioli, L.; Masutti, F.; Passalacqua, M.; Castiglione, A.; Tiribelli, C. The fatty liver index: A simple and accurate predictor of hepatic steatosis in the general population. BMC Gastroenterol. 2006, 6, 33. [CrossRef] [PubMed]

37. Abenavoli, L.; Beaugrand, M. Transient elastography in non-alcoholic fatty liver disease. Ann. Hepatol. 2012, 11, 172-178. [PubMed]

38. Berzigotti, A. Non-invasive assessment of non-alcoholic fatty liver disease: Ultrasound and transient elastography. Rev. Recent Clin. Trials 2014, 9, 170-177. [CrossRef] [PubMed]

39. Takahashi, Y.; Sugimoto, K.; Inui, H.; Fukusato, T. Current pharmacological therapies for nonalcoholic fatty liver disease/nonalcoholic steatohepatitis. World J. Gastroenterol. 2015, 21, 3777-3785. [CrossRef] [PubMed]

40. Mikolasevic, I.; Milic, S.; Turk Wensveen, T.; Grgic, I.; Jakopcic, I.; Stimac, D.; Wensveen, F.; Orlic, L. Nonalcoholic fatty liver disease-A multisystem disease? World J. Gastroenterol. 2016, 22, 9488-9505. [CrossRef] [PubMed] 
41. Abenavoli, L.; Peta, V. Role of adipokines and cytokines in non-alcoholic fatty liver disease. Rev. Recent Clin. Trials 2014, 9, 134-140. [CrossRef] [PubMed]

42. Di Daniele, N.; Noce, A.; Vidiri, M.F.; Moriconi, E.; Marrone, G.; Annicchiarico-Petruzzelli, M.; D’Urso, G.; Tesauro, M.; Rovella, V.; De Lorenzo, A. Impact of Mediterranean diet on metabolic syndrome, cancer and longevity. Oncotarget 2017, 8, 8947-8979. [CrossRef] [PubMed]

43. Papamiltiadous, E.S.; Roberts, S.K.; Nicoll, A.J.; Ryan, M.C.; Itsiopoulos, C.; Salim, A.; Tierney, A.C. A randomised controlled trial of a Mediterranean dietary intervention for adults with non-alcoholic fatty liver disease (MEDINA): Study protocol. BMC Gastroenterol. 2016, 16, 14. [CrossRef] [PubMed]

44. Garcia, M.; Bihuniak, J.D.; Shook, J.; Kenny, A.; Kerstetter, J.; Huedo-Medina, T.B. The effect of the traditional Mediterranean-style diet on metabolic risk factors: A meta-analysis. Nutrients 2016, 8, 168. [CrossRef] [PubMed]

45. Ganesh, S.; Rustgi, V.K. Current pharmacologic therapy for nonalcoholic fatty liver disease. Clin. Liver Dis. 2016, 20, 351-364. [CrossRef] [PubMed]

46. Hsu, W.F.; Sheen, L.Y.; Lin, H.J.; Chang, H.H. A review of western and traditional chinese medical approaches to managing nonalcoholic fatty liver disease. Evid.-Based Complement. Altern. Med. 2016, 2016, 6491420. [CrossRef] [PubMed]

47. Liu, Z.L.; Xie, L.Z.; Zhu, J.; Li, G.Q.; Grant, S.J.; Liu, J.P. Herbal medicines for fatty liver diseases. Cochrane Database Syst. Rev. 2013, CD009059. [CrossRef]

48. Abenavoli, L.; Capasso, R.; Milic, N.; Capasso, F. Milk thistle in liver diseases: Past, present, future. Phytother. Res. 2010, 24, 1423-1432. [CrossRef] [PubMed]

49. Federico, A.; Dallio, M.; Loguercio, C. Silymarin/silybin and chronic liver disease: A marriage of many years. Molecules 2017, 22, 191. [CrossRef] [PubMed]

50. Federico, A.; Trappoliere, M.; Tuccillo, C.; de Sio, I.; Di Leva, A.; Del Vecchio Blanco, C.; Loguercio, C. A new silybin-vitamin E-phospholipid complex improves insulin resistance and liver damage in patients with non-alcoholic fatty liver disease: Preliminary observations. Gut 2006, 55, 901-902. [CrossRef] [PubMed]

51. Aller, R.; Izaola, O.; Gómez, S.; Tafur, C.; González, G.; Berroa, E.; Mora, N.; González, J.M.; de Luis, D.A. Effect of silymarin plus vitamin $\mathrm{E}$ in patients with non-alcoholic fatty liver disease. A randomized clinical pilot study. Eur. Rev. Med. Pharmacol. Sci. 2015, 19, 3118-3124. [PubMed]

52. Loguercio, C.; Andreone, P.; Brisc, C.; Brisc, M.C.; Bugianesi, E.; Chiaramonte, M.; Cursaro, C.; Danila, M.; de Sio, I.; Floreani, A.; et al. Silybin combined with phosphatidylcholine and vitamin E in patients with nonalcoholic fatty liver disease: A randomized controlled trial. Free Radic. Biol. Med. 2012, 52, 1658-1665. [CrossRef] [PubMed]

53. Abenavoli, L.; Greco, M.; Nazionale, I.; Peta, V.; Milic, N.; Accattato, F.; Foti, D.; Gulletta, E.; Luzza, F. Effects of Mediterranean diet supplemented with silybin-vitamin E-phospholipid complex in overweight patients with non-alcoholic fatty liver disease. Expert Rev. Gastroenterol. Hepatol. 2015, 9, 519-527. [CrossRef] [PubMed]

54. Wan, C.W.; Wong, C.N.; Pin, W.K.; Wong, M.H.; Kwok, C.Y.; Chan, R.Y.; Yu, P.H.; Chan, S.W. Chlorogenic acid exhibits cholesterol lowering and fatty liver attenuating properties by up-regulating the gene expression of PPAR- $\alpha$ in hypercholesterolemic rats induced with a high-cholesterol diet. Phytother. Res. 2013, 27, 545-551. [CrossRef] [PubMed]

55. Orhan, I.E.; Şener, B.; Musharraf, S.G. Antioxidant and hepatoprotective activity appraisal of four selected Fumaria species and their total phenol and flavonoid quantities. Exp. Toxicol. Pathol. 2012, 64, 205-209. [CrossRef] [PubMed]

56. Pacana, T.; Cazanave, S.; Verdianelli, A.; Patel, V.; Min, H.K.; Mirshahi, F.; Quinlivan, E.; Sanyal, A.J. Dysregulated hepatic methionine metabolism drives homocysteine elevation in diet-induced nonalcoholic fatty liver disease. PLoS ONE 2015, 10, e0136822. [CrossRef] [PubMed]

57. Mardinoglu, A.; Bjornson, E.; Zhang, C.; Klevstig, M.; Söderlund, S.; Ståhlman, M.; Adiels, M.; Hakkarainen, A.; Lundbom, N.; Kilicarslan, M.; et al. Personal model-assisted identification of NAD+ and glutathione metabolism as intervention target in NAFLD. Mol. Syst. Biol. 2017, 13, 916. [CrossRef] [PubMed]

(C) 2017 by the authors. Licensee MDPI, Basel, Switzerland. This article is an open access article distributed under the terms and conditions of the Creative Commons Attribution (CC BY) license (http:/ / creativecommons.org/licenses/by/4.0/). 